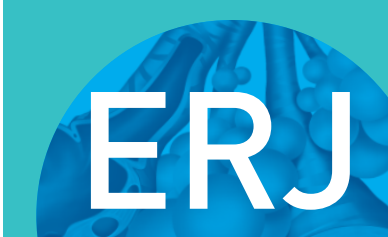

open research

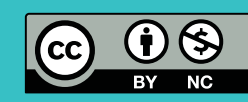

\section{TLR3/TAK1 signalling regulates rhinovirus-induced interleukin-33 in bronchial smooth muscle cells}

\author{
Sangeetha Ramu1,4, Jenny Calvén ${ }^{1,4}$, Charalambos Michaeloudes (10) \\ Mandy Menzel ${ }^{1}$, Hamid Akbarshahi (i] ${ }^{1,3}$, Kian Fan Chung $^{2}$ and Lena Uller ${ }^{1}$
}

Affiliations: ${ }^{1}$ Dept of Experimental Medical Science, Lund University, Lund, Sweden. ${ }^{2}$ Airway Disease section, National Heart and Lung Institute, Imperial College London, London, UK. ${ }^{3}$ Dept of Respiratory Medicine and Allergology, Lund University, Lund, Sweden. ${ }^{4}$ These authors contributed equally.

Correspondence: Lena Uller, Dept of Experimental Medical Science, Unit of Respiratory Immunopharmacology, Lund University, BMC D12, 22184 Lund, Sweden. E-mail: lena.ullerAmed.lu.se

\section{ABSTRACT}

Background: Asthma exacerbations are commonly associated with rhinovirus (RV) infection. Interleukin33 (IL-33) plays an important role during exacerbation by enhancing Type 2 inflammation. Recently we showed that RV infects bronchial smooth muscle cells (BSMCs) triggering production of interferons and IL-33. Here we compared levels of RV-induced IL-33 in BSMCs from healthy and asthmatic subjects, and explored the involvement of pattern-recognition receptors (PRRs) and downstream signalling pathways in IL-33 expression.

Method: BSMCs from healthy and severe and non-severe asthmatic patients were infected with RV1B or stimulated with the PRR agonists poly(I:C) (Toll-like receptor 3 (TLR3)), imiquimod (TLR7) and poly(I:C)/LyoVec (retinoic acid-inducible gene 1 (RIG-I)/melanoma differentiation-associated protein 5 (MDA5)). Knockdown of TLR3, RIG-I and MDA5 was performed, and inhibitors targeting TBK1, nuclear factor- $\kappa \mathrm{B}(\mathrm{NF}-\kappa \mathrm{B})$ and transforming growth factor (TGF)- $\beta$-activated kinase 1 (TAK1) were used. Gene and protein expression were assessed.

Results: RV triggered IL-33 gene and protein expression in BSMCs. BSMCs from patients with non-severe asthma showed higher baseline and RV-induced IL-33 gene expression compared to cells from patients with severe asthma and healthy controls. Furthermore, RV-induced IL-33 expression in BSMCs from healthy and asthmatic individuals was attenuated by knockdown of TLR3. Inhibition of TAK1, but not NF- $\mathrm{KB}$ or TBK1, limited RV-induced IL-33. The cytokine secretion profile showed higher production of IL-33 in BSMCs from patients with non-severe asthma compared to healthy controls upon RV infection. In addition, BSMCs from patients with non-severe asthma had higher levels of RV-induced IL- 8 , TNF- $\alpha$, IL-1 $\beta$, IL-17A, IL-5 and IL-13.

Conclusion: RV infection caused higher levels of IL-33 and increased pro-inflammatory and Type 2 cytokine release in BSMCs from patients with non-severe asthma. RV-induced IL-33 expression was mainly regulated by TLR3 and downstream via TAK1. These signalling molecules represent potential therapeutic targets for treating asthma exacerbations.

@ERSpublications

Rhinovirus-induced IL-33 is overexpressed in bronchial smooth muscle cells from asthmatics, and the production of IL-33 following infection is mediated by activation of TLR3 and downstream TAK1 signalling https://bit.ly/3fXH0h3

Cite this article as: Ramu S, Calvén J, Michaeloudes C, et al. TLR3/TAK1 signalling regulates rhinovirus-induced interleukin-33 in bronchial smooth muscle cells. ERJ Open Res 2020; 6: 001472020 [https://doi.org/10.1183/23120541.00147-2020].

This article has supplementary material available from openres.ersjournals.com

Received: 29 March 2020 | Accepted after revision: 21 June 2020

Copyright $\odot$ ERS 2020. This article is open access and distributed under the terms of the Creative Commons Attribution Non-Commercial Licence 4.0. 


\section{Introduction}

Exacerbations are a major cause of disease morbidity and mortality in asthma, and they have been associated with increased loss of lung function in asthma [1]. Up to $70 \%$ of exacerbations in adult asthma are associated with respiratory viral infections [2], with rhinovirus (RV) being the most common cause of asthma exacerbations [3]. Viruses can trigger innate immune responses via the activation of Toll-like receptors (TLRs), such as TLR3 and TLR7/8, or the RIG-I-like helicases, melanoma differentiation-associated protein 5 (MDA5) and retinoic acid-inducible gene 1 (RIG-I). TLRs are the major group of receptors that recognise pathogen-associated molecular patterns in bacteria, viruses and fungi [4]. During replication, the RV intermediate double-stranded RNA (dsRNA) is released and sensed by TLR3 and/or RIG-I/MDA5 [5]. Therefore, activation of pattern-recognition receptors (PRRs) in airway epithelial and smooth muscle cells represents a critical factor for promoting airway inflammation during episodes of viral infections [6]. The serine/threonine kinase transforming growth factor (TGF)- $\beta$-activated kinase 1 (TAK1) is a member of the mitogen-activated protein kinase (MAPK) family and has been shown to be a mediator of TLR signalling. TAK1 regulates different responses involved in innate and adaptive immunity and has a major function as a downstream mediator of TLR signalling [7].

Bronchial smooth muscle cells (BSMCs) are the main effector of airway hyper-responsiveness, but they also play a significant role in promoting inflammation in asthma $[8,9]$. We have previously shown that BSMCs express functional TLR3 and RIG-I-like receptors [10], indicating that they might have a role in mediating pathogen-induced exacerbations in airway diseases. BSMCs also exhibit pro-inflammatory and immunomodulatory functions via the release of inflammatory cytokines and the expression of a wide range of cell surface molecules, including integrins and co-stimulatory molecules [11]. We have demonstrated earlier that RV infection of BSMCs increases the expression of interleukin (IL-33) [12], which is a key upstream inflammatory cytokine [13] that has received attention as an important player underlying exacerbations of asthma [14].

IL-33 belongs to the IL-1 cytokine family and promotes Type 2 (T2) inflammation [15, 16]. IL-33 is constitutively expressed by structural cells of the airway wall and is mainly located in the cell nucleus [17]. However, IL-33 is also released into the extracellular space in response to cellular stress, injury or death, functioning either as a cytokine or as an alarmin [18]. IL-33 signals through ST2, which is a member of the Toll/IL-1 receptor superfamily, and regulates pro-inflammatory mediators such as IL-1 $\beta$, TNF- $\alpha$ and IL-6 [19]. IL-33/ST2L signals are involved in the activation of macrophages and mast cells, and in the regulation of T2 cytokine production by innate lymphoid cells [20-22]. Epithelial and smooth muscle cell expression of IL-33 is increased in asthma, thus BSMCs may also contribute to the release of IL-33 into the airway lumen $[23,24]$. We have previously shown that RV infection induces IL-33 mRNA and protein expression in BSMCs from both asthmatic and healthy subjects [12]. In recent studies involving experimental RV inoculation of asthmatic subjects, increased levels of IL-33 have been reported, strongly supporting its role in the pathogenesis of exacerbations $[25,26]$. Although these studies have built a case for a pathogenic role of IL-33 in asthma exacerbated by RV infection, the molecular mechanisms mediating RV-induced IL-33 remain unclear.

In this study, we demonstrate that RV infection of BSMCs from asthmatic donors causes overexpression and release of IL-33 mediated by TLR3 activation and by engagement of a TAK1 downstream signalling pathway. We also observed that BSMCs from subjects with non-severe asthma exhibited higher expression of IL-33 than BSMCs from healthy subjects and donors with severe asthma.

\section{Materials and methods}

\section{Human BSMC culture}

Primary human BSMCs from seven severe and eight non-severe patients with asthma and six healthy donors were cultured from bronchial biopsies, as previously described [27]. The subject characteristics are shown in table 1. Ethical permission was obtained by the National Research Ethics Service Committee London Chelsea (REC reference number: 08/H0708/29). Characterisation of the BSMCs was based on their distinctive "hill and valley" morphology and on positive immunostaining for calponin, smooth muscle $\alpha$-actin and myosin $\mathrm{H}$ chain as previously described [28]. BSMCs were cultured in DMEM with 10\% fetal bovine serum (FBS), $1 \%$ penicillin streptomycin (PEST) and $1 \%$ amphotericin B (Life Technologies, Stockholm, Sweden) in 5\% $\mathrm{CO}_{2}$ at $37^{\circ} \mathrm{C}$. For experiments, BSMCs at passages 3-6 were seeded into 6- or 12-well plates (Nunc, Life Technologies, Carlsbad, CA, USA), and at $80-90 \%$ confluence the growth medium was replaced with DMEM containing reduced FBS (1\%) for $24 \mathrm{~h}$ prior to all experiments.

\section{Infection with rhinovirus $1 B$}

The minor group rhinovirus RV1B was grown in Ohio HeLa cells (European Collection of Cell Cultures) as previously described [29] and was obtained from clarified cell lysates $\left(1.58 \times 10^{7}\right.$ tissue culture infectious 


\begin{tabular}{|c|c|c|c|}
\hline & Healthy controls & Non-severe asthma & Severe asthma \\
\hline Subjects $n$ & $6^{\#}$ & 8 & $7^{\Uparrow}$ \\
\hline Sex male/female $n$ & $3 / 1$ & $4 / 4$ & $5 / 2$ \\
\hline Age years & $60.00(53-65)$ & $47.00(27-67)$ & $41.00(26-55)$ \\
\hline Smoking yes/no/former $\mathrm{n}$ & $0 / 4 / 0$ & $0 / 6 / 2$ & $0 / 6 / 0$ \\
\hline FEV $1 \%$ pred & $97.5(84-101)$ & $83.55(63.20-90.00)$ & 73.80 (62.40-98) \\
\hline $\mathrm{FEV}_{1} / \mathrm{FVC}$ ratio $\%$ & $72.75(70-84)$ & $73.37(50.55-82.59)$ & $71.67(56.58-79.30)$ \\
\hline BDP equivalent & 0 & $1500(100.0-1600)$ & $1600(1000-2000)^{*}$ \\
\hline $\mathrm{PC}_{20}$ & NA & $1.016(0.35-6.27)$ & $1.255(0.89-1.61)$ \\
\hline OCS mg & No & No & Yes (4) \\
\hline \multicolumn{4}{|c|}{$\begin{array}{l}\text { Data are presented as median (range), unless otherwise stated. } \mathrm{FEV}_{1} \text { : forced expiratory volume in } 1 \mathrm{~s} \text {; } \\
\% \text { pred: \% predicted; FVC: forced vital capacity; BDP: beclometasone dipropionate; } \mathrm{PC}_{20} \text { : the provocative } \\
\text { concentration of methacholine that results in a } 20 \% \text { drop in } \mathrm{FEV}_{1} \text {; OCS: oral corticosteroid; NA: } \\
\text { non-applicable. \#: patient characteristics missing from two healthy donors; } \text { : patient characteristics } \\
\text { missing from one donor with severe asthma. *: } \mathrm{p}<0.05 \text { compared to patients with non-severe asthma. }\end{array}$} \\
\hline
\end{tabular}

dose (TCID50)/mL). Based on our previous studies we chose an optimal concentration of RV for the infection experiments $[10,12]$. BSMCs were infected with RV1B at 1 multiplicity of infection (MOI) for $1 \mathrm{~h}$ at room temperature while shaking. Thereafter, the inoculum was removed and replaced with DMEM containing 1\% FBS. In further experiments, BSMCs were stimulated with different PRR agonists such as TLR3 agonist poly(I:C) $\left(10 \mu \mathrm{g} \cdot \mathrm{mL}^{-1}\right)$, TLR7 agonist imiquimod $\left(10 \mu \mathrm{g} \cdot \mathrm{mL}^{-1}\right)$ and RIG-I/MDA5 agonist poly(I:C)/LyoVec $\left(0.5 \mu \mathrm{g} \cdot \mathrm{mL}^{-1}\right)$. Cell lysates and supernatants were collected $24 \mathrm{~h}$ post-infection, respectively, and utilised for gene and protein expression analysis, respectively.

\section{Transfection with siRNA}

BSMCs were transfected with small interfering RNA (siRNA) targeted against TLR3 (ID: s235), MDA5 (ID: s125361) or RIG-I (ID: s223616) or with nonspecific siRNA (Ambion, Thermo Scientific, Waltham, MA, USA) at a concentration of $10 \mathrm{nM}$ using Lipofectamine RNAiMAX (Ambion) as a transfection agent. The culture medium was replaced $24 \mathrm{~h}$ after transfection. After $48 \mathrm{~h}$ from transfection, BSMCs were infected with 1 MOI RV1B for $24 \mathrm{~h}$.

\section{Pharmacological inhibitors}

Pharmacological synthetic inhibitors were used to block the proteins that regulate interferon regulatory factors (IRFs) and NF-kB downstream signalling pathways. The TBK1-mediated activation of IRF3 was blocked by $0.1 \mu \mathrm{M}$ BX795 [30], IkB kinase (IKK)-mediated NF-kB activation by $10 \mathrm{nM}$ PS1145 [31] and TAK1 activation by $0.1 \mu \mathrm{M}$ 5Z-7-oxozeaenol [32] (Sigma-Aldrich, Stockholm, Sweden). The cells were incubated at $37^{\circ} \mathrm{C}$ for $1 \mathrm{~h}$ in the presence of all inhibitors and then infected with 1 MOI RV1B for $24 \mathrm{~h}$.

\section{RNA extraction and quantification of gene expression by real-time quantitative PCR}

Total RNA was extracted from BSMCs using an RNA extraction kit (Nucleospin ${ }^{\circledR}$ RNA II, Macherey-Nagel, Düren, Germany). Then $1 \mu \mathrm{g}$ of RNA was reverse-transcribed to cDNA (Precision Nanoscript Reverse Transcription Kit, PrimerDesign, Southampton, UK) and real-time quantitative PCR (RT-qPCR) was performed. In brief, a Mx3005P qPCR system (Stratagene, La Jolla, CA, USA) with standard cycling parameters was used to perform thermo-cycling and real-time detection of PCR products. The following primer sequences (PrimerDesign, Southampton, UK) were used: human IL-33: AAAGAAAGATAAGGTGTTACTGAGTTA (forward) and GCAACCAGAAGTCTTTTGTAGG (reverse), human RIG-I: TTCTCTTGATGCGTCAGTGATA (forward) and CCGTGATTCCACTTTCCTGAA (reverse), human MDA5: GTCTCGTCACCAATGAAATAGC (forward) and TTATACATCATCTTCT CTCGGAAATC (reverse), human TLR3: GTGTGAAAGTATTGCCTGGTTTGT (forward) and ATGATAGTGAGGTGGAGTGTTGC (reverse). Target genes were normalised to the geometric mean of two reference genes, ubiquitin $\mathrm{c}$ (UBC) and glyceraldehyde 3-phosphate dehydrogenase (GAPDH), and relative gene expression compared to control was analysed by the ${ }^{\Delta \Delta} \mathrm{Ct}$ method [33]. Groups were normalised to untreated control or RV1B when appropriate.

\section{Western blot analysis}

Protein expression of IL-33 was quantified by Western blot. Experiments were performed as described in previous sections and cells were lysed $24 \mathrm{~h}$ post-RV1B-infection using a lysis buffer containing $1 \%$ 
TritonX-100, $10 \mathrm{mM}$ Tris- $\mathrm{HCl}, 50 \mathrm{mM} \mathrm{NaCl}, 5 \mathrm{mM}$ EDTA, $30 \mathrm{mM}$ Na4P2O7, $50 \mathrm{mM} \mathrm{NaF}, 0.1 \mathrm{mM}$ Na3VO4, $1 \%$ phosphatase and protease inhibitors (Sigma-Aldrich). Protein concentrations were determined by BCA protein assay (Pierce Thermo Scientific, Waltham, MA, USA) for each sample, and equal amounts of protein were loaded and electrophoresed on a 10\% TGX stain-free gel (Bio-Rad Laboratories AB, Solna, Sweden), before blotting on a Trans-Blot Turbo Transfer System (Bio-Rad Laboratories $A B)$. This was followed by blocking of the membrane in $5 \%(w / v)$ milk in Tris-buffered saline Tween-20 and overnight incubation at $4{ }^{\circ} \mathrm{C}$ with primary antibodies TLR3, MDA5 and RIG-I (Cell Signalling Technology, Leiden, The Netherlands); anti-IL-33 antibody Nessy-1 clone (N1) (Enzo Life Sciences, Exeter, UK). Then the membrane was washed and incubated for $1 \mathrm{~h}$ with secondary antibodies (anti-mouse IgG HRP-linked Ab; Cell signalling Technology). Detection was performed by chemiluminescence using SuperSignal West FEMTO substrate (Pierce Thermo Scientific) and immunoblots were visualised by LI-COR Odyssey Fc Imager (LI-COR Biosciences, Lincoln, NE, USA) and Image Studio (v.3.1.4; LI-COR Sciences).

\section{Protein quantification by multiplex ELISA}

Cytokine levels were measured in cell-free supernatants by Luminex immunoassay according to the manufacturer's instructions (R\&D Systems, Abingdon, UK). Data were acquired on a calibrated and validated Luminex MAGPIX instrument (R\&D Systems) as per manufacturer's instructions.

\section{Statistical analysis}

Data were analysed using the GraphPad Prism 7 software (GraphPad Software). Differences between groups were analysed using the Mann-Whitney U test or Kruskal-Wallis test with post hoc analysis. Data are presented as either median or mean \pm SEM. Values were considered significantly different at a $\mathrm{p}$-value of $<0.05$.

\section{Results}

IL-33 expression is increased in BSMCs from patients with non-severe asthma compared to cells from healthy patients and those with severe asthma during RV infection

We demonstrated an increased baseline IL-33 expression in BSMCs from patients with non-severe asthma compared to healthy donors (supplementary figure S1). Furthermore, RV-induced IL-33 expression in BSMCs from healthy controls as well as from the two groups of asthmatic patients, non-severe and severe asthma (figure 1a and b). BSMCs from non-severe asthmatic patients displayed a significantly higher
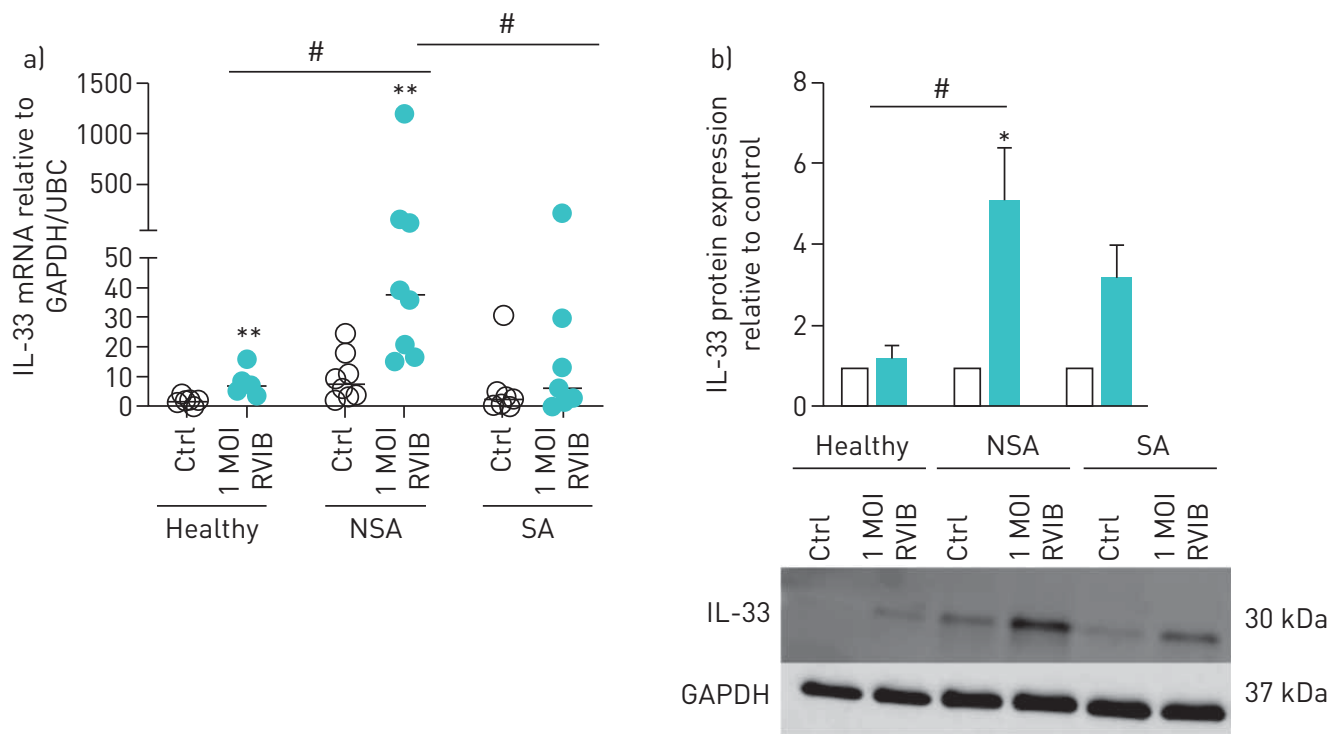

FIGURE 1 Bronchial smooth muscle cells (BSMCs) from healthy and asthmatic subjects were infected with 1 multiplicity of infection (MOI) RV1B. a) Gene expression of interleukin (IL)-33 in healthy subjects (n=6) and those with non-severe asthma (NSA; $n=8$ ) and severe asthma (SA: $n=7)$; data are presented as median fold change of healthy control relative to glyceraldehyde 3-phosphate dehydrogenase (GAPDH)/ubiquitin c (UBC) gene expression. b) Protein expression of IL-33 healthy subjects (n=3) and those with non-severe asthma $(n=6)$ and severe asthma $(n=6)$; data are presented as mean \pm SEM relative to GAPDH protein expression. *: $p<0.05 ;{ }^{* *}: \quad p<0.01$ versus control (Mann-Whitney $U$ test); \#: $p<0.01$ versus RV1B between groups (Kruskal-Wallis test and Dunn's multiple comparison post hoc test). 
induction of IL-33 gene expression (figure 1a) as well as IL-33 protein expression (figure 1b) compared to BSMCs from healthy patients and those with severe asthma in response to RV infection.

\section{Activation of TLR3 and RIG-I-like receptors, but not TLR7, induces IL-33 expression in BSMCS}

We have previously demonstrated that BSMCs, similar to bronchial epithelial cells express TLR3 and RIG-I-like receptors and that activation of these receptors may be critically involved in IFN production [10]. Here we confirmed and expanded the prior observations demonstrating the involvement of specific PRRs in mediating IL-33 expression in BSMCs. BSMCs were stimulated with the TLR3 agonist poly(I:C), the RIG-I/MDA5 agonist poly(I:C)/LyoVec or the TLR7 agonist imiquimod for $24 \mathrm{~h}$, and IL-33 mRNA expression was measured. We found that IL-33 expression in BSMCs was markedly induced by stimulation of TLR3 with poly(I:C), and marginally increased by stimulation with poly(I:C)/LyoVec (shown to be specific for RIG-I-like receptors), but not by stimulation with a TLR7 ligand. This response pattern was similar in BSMCs from healthy (figure 2a), non-severe (figure $2 \mathrm{~b}$ ) and severe asthmatic subjects (figure 2c). We further confirmed that poly(I:C) and, to various extents, poly(I:C)/LyoVec increased IL-33 protein expression in BSMCs from healthy (figure $2 \mathrm{~d}$ ) and non-severe (figure $2 \mathrm{e}$ ) and severe patients with asthma (figure 2f).

\section{$R V$-induced expression of IL-33 is attenuated by knockdown of TLR3, but not of RIG-I-like receptors in BSMCs from healthy and asthmatic patients}

To more specifically address the involvement of TLR3 and RIG-I-like receptors in RV-mediated IL-33 expression, we knocked down the receptors TLR3, MDA5 and RIG-I using siRNA. BSMCs were transfected with siRNA sequences specific for the target genes or control scramble siRNA. Efficient knockdown was confirmed by RT-qPCR and Western blot demonstrating that both mRNA and protein expression were reduced to $<10 \%$ of their basal levels (supplementary figure S2). Our results further showed that knockdown of TLR3 attenuated RV-induced IL-33 mRNA (figure 3a-c) and protein expression (figure 3d-f) in BSMCs from both healthy and asthmatic patients. Knockdown of RIG-I-like receptors did not reduce the RV-induced IL-33 in BSMCs. These results also confirmed that the TLR3 pathway is involved in RV-induced IL-33 gene and protein expression in BSMCs to a similar extent in healthy and asthmatic subjects.

\section{Inhibition of TAK1 reduces RV-induced IL-33 expression in BSMCs from both asthmatic and} healthy subjects

Next, we addressed the involvement of TLR3 downstream signalling pathways in the enhanced effect of RV1B on IL-33 expression in BSMCs. TLRs recruit and signal through adaptor molecules, leading to activation of the transcription factors IRF3/7, AP-1 and NF-kB, which regulate immune responses [34].
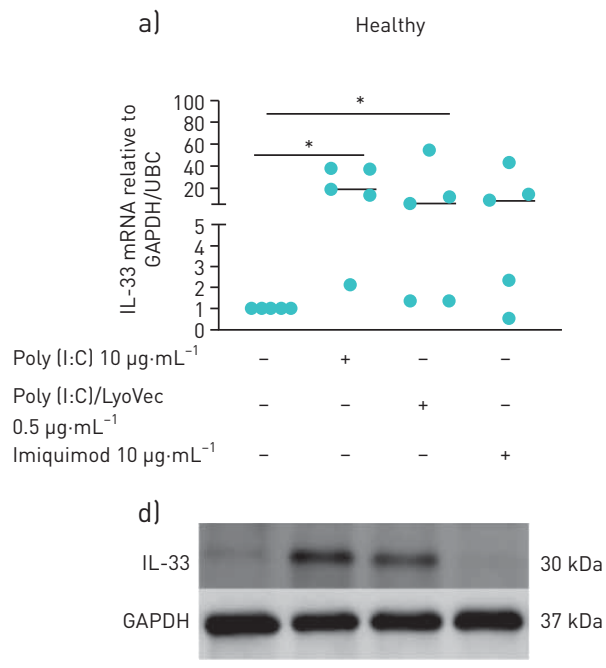

b)

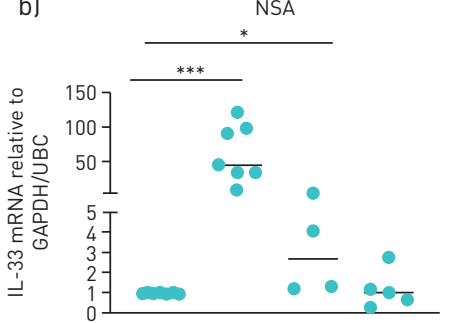

Poly (I:C) $10 \mu \mathrm{g} \cdot \mathrm{mL}^{-1}$ Poly (I:C)/LyoVec $0.5 \mu \mathrm{g} \cdot \mathrm{mL}^{-1}$ Imiquimod $10 \mu \mathrm{g} \cdot \mathrm{mL}^{-1}$

e)

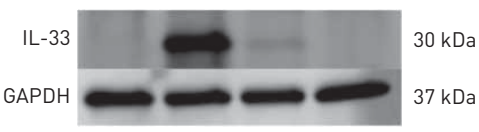

c)

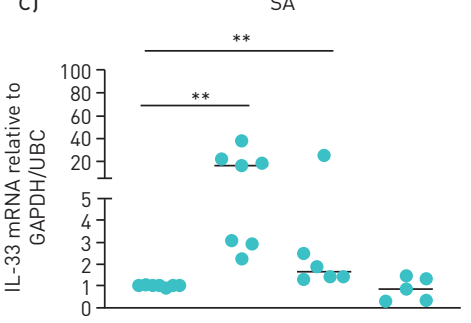

Poly (I:C) $10 \mu \mathrm{g} \cdot \mathrm{mL}^{-1}$ Poly (I:C)/LyoVec $0.5 \mu \mathrm{g} \cdot \mathrm{mL}^{-1}$ Imiquimod $10 \mu \mathrm{g} \cdot \mathrm{mL}^{-1} \quad-\quad+\quad-\quad+$

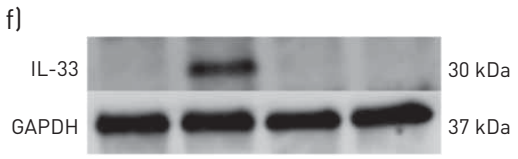

FIGURE 2 Bronchial smooth muscle cells (BSMCs) were stimulated with the Toll-like receptor 3 (TLR3) agonist poly(l:C) (10 $\mu \mathrm{g} \cdot \mathrm{mL}^{-1}$ ), the retinoic acid-inducible gene 1 (RIG-I)/ melanoma differentiation-associated protein 5 (MDA5) agonist poly(l:C)/LyoVec (0.5 $\mu \mathrm{g} \cdot \mathrm{mL}^{-1}$ ) or the TLR7 agonist imiquimod $\left(10 \mu \mathrm{g} \cdot \mathrm{mL}^{-1}\right)$ for $24 \mathrm{~h}$. Gene expression of interleukin (IL)-33 in a) healthy subjects (n=5), b) patients with non-severe asthma (NSA; $\mathrm{n}=4-7)$ and c) patients with severe asthma $(S A ; n=6)$; data are presented as median fold change of control relative to ubiquitin $c$ (UBC)/ glyceraldehyde 3-phosphate dehydrogenase (GAPDH) expression. A representative Western blot of IL-33 protein expression in d) healthy subjects, e) patients with non-severe asthma and f) patients with severe asthma. Comparison of different groups was performed using the Mann-Whitney $U$ test. ${ }^{*}: p<0.05 ;{ }^{* *}: p<0.01 ;{ }^{* *}: p<0.001$ versus control. 


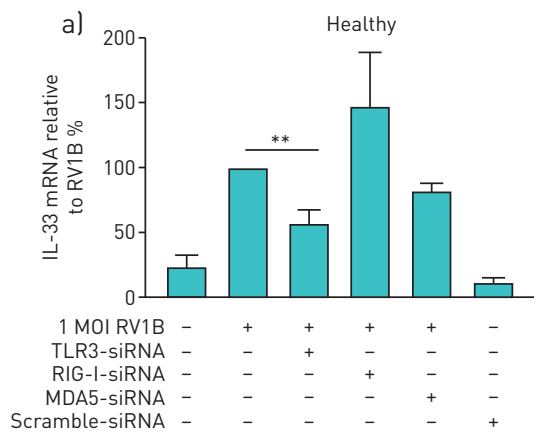

d)

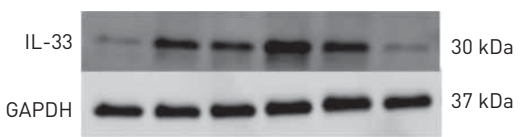

b)

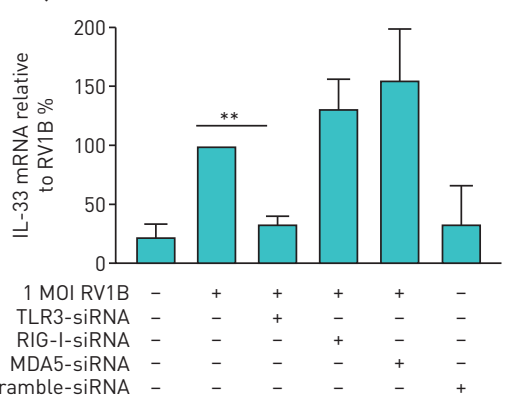

e)

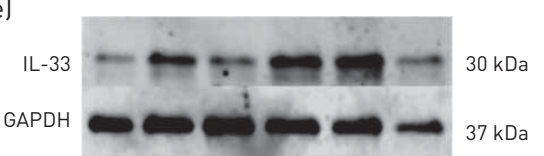

c)

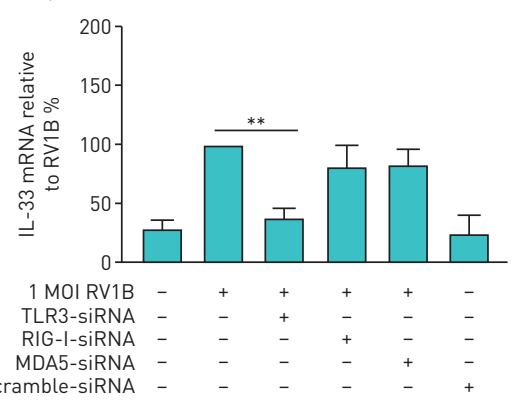

f)

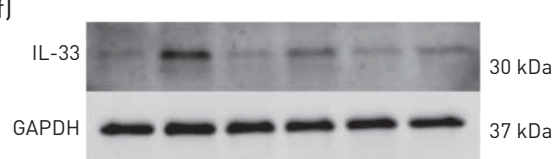

FIGURE 3 Bronchial smooth muscle cells (BSMCs) from healthy and asthmatic subjects were transfected with siRNA specific for Toll-like receptor 3 (TLR3) (10 nM), retinoic acid-inducible gene 1 (RIG-I) (10 nM) or melanoma differentiation-associated protein 5 (MDA5) (10 nM) for $48 \mathrm{~h}$ prior to infection with RV1B. Gene expression of interleukin (IL)-33 in a) healthy subjects ( $n=6), b$ ) patients with non-severe asthma (NSA; $n=7$ ) and c) patients with severe asthma (SA; $n=7)$; data are presented as percentage relative to RV1B expression. A representative Western blot of IL-33 protein expression in $d$ ) healthy subjects $(n=3)$, e) patients with non-severe asthma $(n=3-5)$ and $f$ ) patients with severe asthma ( $n=3)$. Comparison of different groups was performed using the Mann-Whitney $U$ test. ${ }^{* *}: p<0.01$ versus RV1B.

BSMCs were thus pretreated with BX795, PS1145 and 5Z-7-oxozeaenol respectively, followed by infection with RV1B for $24 \mathrm{~h}$. Pretreatment with the TAK1 inhibitor 5Z-7-oxozeaenol reduced RV-induced IL-33 gene (figure $4 \mathrm{a}-\mathrm{c}$ ) and protein expression (figure $4 \mathrm{~d}-\mathrm{f}$ ) in BSMCs from both healthy and asthmatic subjects. However, pretreatment with TBK1 and IKK $\beta$ inhibitors did not reduce RV-induced IL-33 expression in BSMCs (figure $4 \mathrm{a}-\mathrm{f}$ ). Together with the knockdown data, this finding suggests that RV-induced IL-33 expression is regulated by TLR3 activation and the TAK1 signalling pathway in BSMCs.

\section{Heat-map representation of $R V$-induced cytokine release from BSMCs in both healthy and asthmatic subjects}

We next investigated whether RV induced asthma-related cytokine release in cell culture supernatants from BSMCs. Since we found higher levels of RV-induced IL-33 expression in patients with non-severe asthma, we only performed cytokine analysis in healthy individuals and those with non-severe asthma. We observed that IL-33 release by BSMCs was higher in patients with non-severe asthma compared to healthy controls (figure 5), confirming our finding at the gene and intracellular protein level (figure $1 \mathrm{a}$ and $\mathrm{b}$ ). We also observed that RV-induced IFN- $\beta$ secretion was lower in BSMCs from patients with non-severe asthma compared to healthy controls. Further, RV-induced protein levels of IL-13, IL-5, IL-17A, IL-8, TNF- $\alpha$ and IL- $1 \beta$ were higher in BSMCs from patients with non-severe asthma compared to healthy controls (figure 5).

\section{Discussion}

We have previously shown that BSMCs produce IFNs upon viral infection, suggesting that these cells may contribute to host defence in bronchial RV infections [10]. In this study, we showed that IL-33 expression is increased at baseline in BSMCs from non-severe asthmatic subjects compared to healthy donors (supplementary figure S1). This observation is partly in agreement with a previous report by PREFONTAINE et al. [23] describing elevated levels of IL-33 in moderate asthma compared to healthy controls in bronchial epithelial cells. In a recent study, we observed that RV triggered IL-33 expression in BSMCs from healthy individuals in a replication-dependent manner with additional preliminary observations of similar effects in BSMCs from three asthmatic donors [12]. Importantly, as a response to RV infection, we now demonstrate overexpression of IL-33 gene and protein in BSMCs from patients with non-severe asthma compared to cells from healthy donors and patients with severe asthma (figure 1). We also confirmed that RV-induced IL-33 release was higher in BSMCs from patients with non-severe asthma compared to control cells (figure 5). Further, we explored the involvement of PRRs and downstream signalling pathways by demonstrating that RV1B increases the expression of IL-33 in BSMCs via TLR3-mediated activation of the TAK1 signalling cascade (figure 6).

Our data indicate that less IL-33 is induced in patients with severe asthma compared to those with non-severe asthma even after RV infection. It has previously been shown that dexamethasone readily inhibits RV-induced IL-33 in BSMCs [12] indicating that the reduced expression in BSMCs from patients with severe 

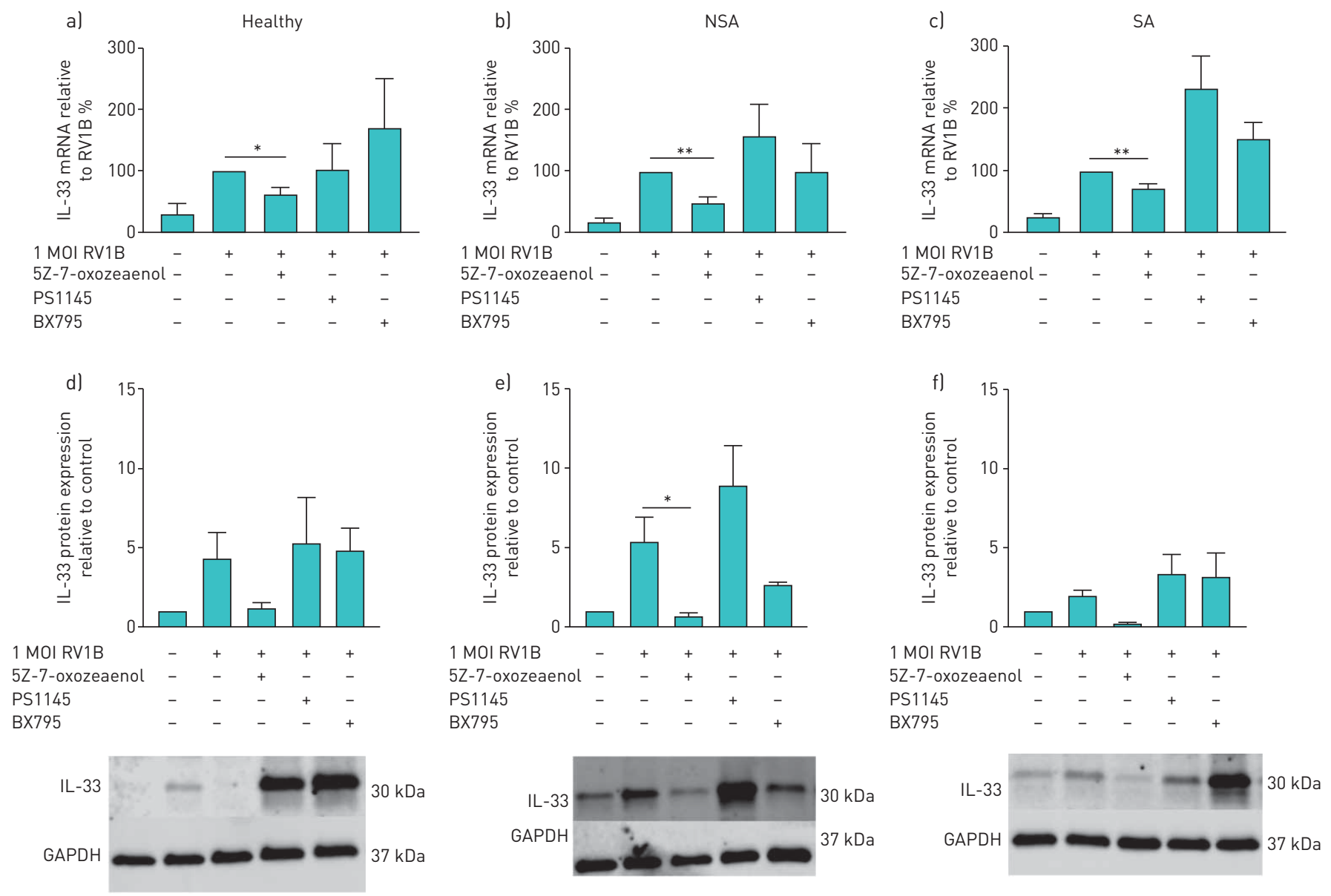

FIGURE 4 Bronchial smooth muscle cells (BSMCs) from healthy and asthmatic subjects were pretreated with specific inhibitors for transforming growth factor (TGF)- $\beta$-activated kinase 1 (TAK1), (5Z-7-oxozeaenol, $0.1 \mu \mathrm{M})$, IKK $\beta$ (PS1145, $10 \mu \mathrm{M}$ ) or TBK1/IKK $\varepsilon$ (BX795, $0.1 \mu \mathrm{M})$ for $1 \mathrm{~h}$ prior to infection with RV1B. Gene expression of interleukin (IL)-33 in a) healthy subjects ( $=5$ ), b) patients with non-severe asthma (NSA; $n=7$ ) and c) patients with severe asthma (SA; $n=7)$; data are presented as percentage relative to RV1B expression. Protein expression of IL-33 in d) healthy subjects $(n=3)$, e) patients with non-severe asthma $(n=3-5)$ and f) patients with severe asthma ( $n=3)$; data are presented as mean \pm SEM relative to glyceraldehyde 3-phosphate dehydrogenase (GAPDH) protein expression. Comparison of different groups was performed using the Mann-Whitney U test. *: $p<0.05,{ }^{* *}$ : $p<0.01$ versus RV1B.

asthma may be due to increased corticosteroid usage. Our data are in line with findings by CHANG et al. [24] showing that BSMCs from patients with severe asthma, most of which received maintenance treatment with oral steroids as in this study, released less CCL11 in response to TNF- $\alpha$ than those from patients with non-severe asthma. In contrast, PREFONTAINE et al. [35] reported no difference in the baseline expression of IL-33 in BSMCs from patients with non-severe asthma and those with severe asthma. The discrepancy between the two studies could be due to differences in corticosteroid treatment among the subjects; however, although the subjects of this study were also on oral and/or high doses of inhaled corticosteroids, there is no information on the doses used. Hence, there is a possibility that systemic corticosteroid treatment, commonly prescribed in severe asthma and regularly used by most patients with severe asthma recruited in this study, could have reduced the IL-33 responsiveness of the BSMCs. Our observations would support the idea of looking for clinical efficacy of IL-33 anti-biologics with the aim of reducing the need for systemic steroids, a treatment with adverse side effects, rather than offering novel efficacy features.

RV has been reported to activate not only TLR3, but also the RIG-I-like receptors in BSMCs [10]. Using specific ligands and knockdown approaches, we confirmed the role of TLR3 in the regulation of IL-33. However, based on our data, RIG-I and MDA5 appear not to be involved in RV-induced IL-33 expression in BSMCs.

Intriguingly, by using known inhibitors of molecules potentially involved in downstream signalling pathways, we further demonstrated that TAK1, but neither NF-kB nor TBK1, was involved in RV infection-induced expression of IL-33 in BSMCs. A previous report demonstrated that TAK1 plays a 


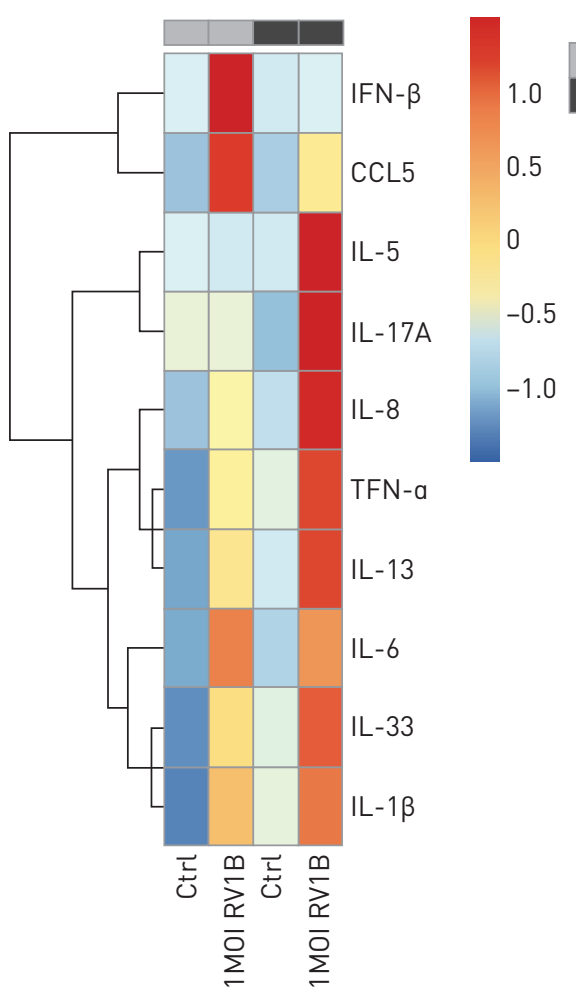

FIGURE 5 Bronchial smooth muscle cells (BSMCs) from healthy and asthmatic subjects were infected with 1 multiplicity of infection (MOI) RV1B. Cell-free supernatants were obtained $24 \mathrm{~h}$ post-rhinovirus (RV)-infection. Cytokine levels were measured by multiplex ELISA and expressed as net median fluorescent intensity (MFI). Data were obtained from six healthy and eight subjects with non-severe asthma.

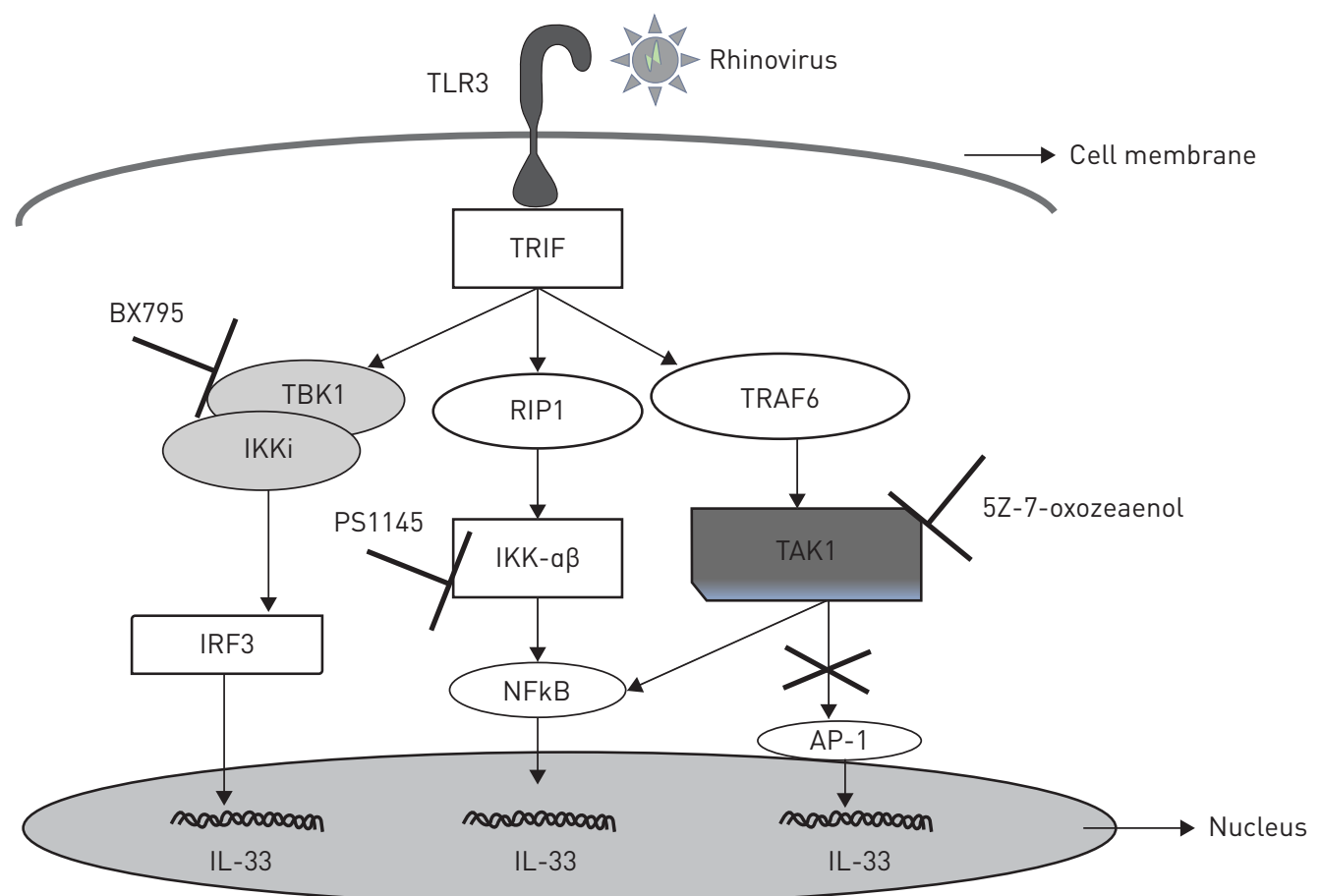

FIGURE 6 Proposed signalling mechanism involved in rhinovirus-induced interleukin (IL)-33 expression in bronchial smooth muscle cells (BSMCs). Recognition of RV1B by Toll-like receptor 3 (TLR3) leads to the recruitment of adaptor molecules that activate transforming growth factor (TGF)- $\beta$-activated kinase 1 (TAK1). Activation of TAK1 results in the activation of transcription factors that increase rhinovirus (RV)-induced IL-33 expression in BSMCs. 
critical role in the pathogenesis of airway inflammation $[29,36]$. TAK1 has been shown to activate NF-kB and MAP kinase pathways, resulting in production of pro-inflammatory cytokines [37] and in airway remodelling through the enhancement of growth factor-induced proliferation of airway smooth muscle cells [38]. Here we reported that RV-induced IL-33 expression in BSMCs is mediated by TAK1, suggesting that TAK1 is a novel therapeutic target for viral-induced airway inflammation. Further investigations are required to elucidate the importance of this pathway.

One limitation to using pharmacological inhibitors for studying involvement of specific proteins in cellular pathways is nonspecific targeting, especially those targeting protein kinases. Therefore, we cannot strictly rule out the possibility that the inhibitors used in our study may have acted through other signalling proteins than those investigated. Another unrelated limitation to our study is the age difference between the healthy and asthmatic subjects, with the healthy subjects being considerably older. Although we cannot exclude that the capacity of BSMCs to produce IL-33 in response to RV infection may be influenced by age, we have not found any evidence in the literature to support variations in innate immune responses to RV in sole relation to age in the adult population. This will need further investigation in a separate study.

The present findings are of interest in view of the potential pathogenic roles of IL-33 and with the consideration that BSMCs might be a major source of this cytokine in asthma. Indeed, discrepancy between epithelial immunoreactivity and bronchoalveolar lavage fluid levels of IL-33 is such that other sources, notably smooth muscle cells, likely are important contributors to the IL-33 that is released in vivo in asthma [23]. There is significant interest in IL-33 as a target molecule for drugs aimed at treatment of viral infection-evoked asthma exacerbations [39].

In conclusion, we report a novel mechanism of RV-induced IL-33 expression through a TLR3/TAK1 signalling pathway. These signalling molecules may be involved in the pathogenesis of respiratory viral-mediated airway inflammation and could represent potential therapeutic targets for treating viral-induced asthma exacerbations.

Support statement: This study was supported by financial grants from the Swedish Research Council, the Swedish Heart-Lung Foundation and VINNOVA. Funding information for this article has been deposited with the Crossref Funder Registry.

Author contributions: S. Ramu, J. Calvén and L. Uller contributed to the design of the study and supervised the scientific work. S. Ramu, C. Michaeloudes, J. Calvén, M. Menzel, H. Akbarshahi, L. Uller and K.F. Chung contributed to the acquisition, analysis and interpretation of the data. S. Ramu drafted the manuscript, and S. Ramu, C. Michaeloudes, J. Calvén, M. Menzel, H. Akbarshahi, L. Uller and K.F. Chung revised the manuscript. All authors have read and approved the submission of this manuscript.

Conflict of interest: S. Ramu has nothing to disclose. J. Calvén has nothing to disclose. C. Michaeloudes has nothing to disclose. M. Menzel has nothing to disclose. H. Akbarshahi has nothing to disclose. K.F. Chung reports grants and personal fees for an advisory board meeting from GlaxoSmithKline, personal fees for advisory board meetings and speaker bureaus from AstraZeneca and Novartis, grants and personal fees for a speaker bureau from Merck, personal fees for advisory board meetings from Boehringer Ingelheim and TEVA, personal fees for a consulting meeting from Roche, and personal fees for the Scientific Board of The Clean Breathing Institute from GSK Consumer Health Care, all outside the submitted work. L. Uller has nothing to disclose.

\section{References}

1 Dougherty RH, Fahy JV. Acute exacerbations of asthma: epidemiology, biology and the exacerbation-prone phenotype. Clin Exp Allergy 2009; 39: 193-202.

2 Adeli M, El-Shareif T, Hendaus MA. Asthma exacerbation related to viral infections: an up to date summary. J Family Med Prim Care 2019; 8: 2753-2759.

3 Singh AM, Busse WW. Asthma exacerbations. 2: aetiology. Thorax 2006; 61: 809-816.

4 Mogensen TH. Pathogen recognition and inflammatory signaling in innate immune defenses. Clin Microbiol Rev 2009; 22: 240-273.

5 Kalali BN, Kollisch G, Mages J, et al. Double-stranded RNA induces an antiviral defense status in epidermal keratinocytes through TLR3-, PKR-, and MDA5/RIG-I-mediated differential signaling. J Immunol 2008; 181 2694-2704.

6 Jezierska A, Kolosova IA, Verin AD. Toll like receptors signaling pathways as a target for therapeutic interventions. Curr Signal Transduct Ther 2011; 6: 428-440.

7 Irie $\mathrm{T}$, Muta $\mathrm{T}$, Takeshige K. TAK1 mediates an activation signal from toll-like receptor(s) to nuclear factor-kappaB in lipopolysaccharide-stimulated macrophages. FEBS Lett 2000; 467: 160-164.

$8 \mathrm{Du}$ Y, Zhao JH, Li X, et al. Dissociation of FK506-binding protein $12.6 \mathrm{kD}$ from ryanodine receptor in bronchial smooth muscle cells in airway hyperresponsiveness in asthma. Am J Resp Cell Mol 2014; 50: 398-408.

9 Zuyderduyn S, Sukkar MB, Fust A, et al. Treating asthma means treating airway smooth muscle cells. Eur Respir J 2008; 32: 265-274.

10 Calven J, Yudina Y, Uller L. Rhinovirus and dsRNA induce RIG-I-like receptors and expression of interferon beta and lambda1 in human bronchial smooth muscle cells. PLoS One 2013; 8: e62718.

11 Hirst SJ. Regulation of airway smooth muscle cell immunomodulatory function: role in asthma. Respir Physiol Neurobiol 2003; 137: 309-326. 
12 Calven J, Akbarshahi H, Menzel M, et al. Rhinoviral stimuli, epithelial factors and ATP signalling contribute to bronchial smooth muscle production of IL-33. J Transl Med 2015; 13: 281.

13 Chan BCL, Lam CWK, Tam LS, et al. IL33: roles in allergic inflammation and therapeutic perspectives. Front Immunol 2019; 10: 364.

14 Kumar RK, Herbert C. IL-33-dependent type 2 inflammation in asthma exacerbations. Am J Respir Crit Care Med 2015; 191: 237-238.

15 Lin J, Zhang LL, Zhao GQ, et al. A novel interleukin 33/ST2 signaling regulates inflammatory response in human corneal epithelium. PLoS One 2013; 8: e60963.

16 Yu YH, Deng WW, Lei JH. Interleukin-33 promotes Th2 immune responses in infected mice with Schistosoma japonicum. Parasitol Res 2015; 114: 2911-2918.

17 Moussion C, Ortega N, Girard JP. The IL-1-like cytokine IL-33 is constitutively expressed in the nucleus of endothelial cells and epithelial cells in vivo: a novel 'alarmin'? PLoS One 2008; 3: e3331.

18 Cayrol C, Girard JP. IL-33: an alarmin cytokine with crucial roles in innate immunity, inflammation and allergy. Curr Opin Immunol 2014; 31: 31-37.

19 Fattori V, Hohmann MSN, Rossaneis AC, et al. Targeting IL-33/ST2 signaling: regulation of immune function and analgesia. Expert Opin Ther Targets 2017; 21: 1141-1152.

20 Hung LY, Lewkowich IP, Dawson LA, et al. IL-33 drives biphasic IL-13 production for noncanonical Type 2 immunity against hookworms. Proc Natl Acad Sci USA 2013; 110: 282-287.

21 Allakhverdi Z, Smith DE, Comeau MR, et al. Cutting edge: the ST2 ligand IL-33 potently activates and drives maturation of human mast cells. J Immunol 2007; 179: 2051-2054.

22 Joshi AD, Oak SR, Hartigan AJ, et al. Interleukin-33 contributes to both M1 and M2 chemokine marker expression in human macrophages. BMC Immunol 2010; 11: 52.

23 Prefontaine D, Nadigel J, Chouiali F, et al. Increased IL-33 expression by epithelial cells in bronchial asthma. J Allergy Clin Immunol 2010; 125: 752-754.

24 Chang PJ, Bhavsar PK, Michaeloudes C, et al. Corticosteroid insensitivity of chemokine expression in airway smooth muscle of patients with severe asthma. J Allergy Clin Immunol 2012; 130: 877-885. e5.

25 Jackson DJ, Makrinioti H, Rana BM, et al. IL-33-dependent type 2 inflammation during rhinovirus-induced asthma exacerbations in vivo. Am J Respir Crit Care Med 2014; 190: 1373-1382.

26 Southworth T, Pattwell C, Khan N, et al. Increased type 2 inflammation post rhinovirus infection in patients with moderate asthma. Cytokine 2020; 125: 154857.

27 Twort C, Van Breemen C. Human airway smooth muscle in culture. Tissue Cell 1988; 20: 339-344.

28 Issa $\mathrm{R}$, Xie $\mathrm{S}$, Khorasani $\mathrm{N}$, et al. Corticosteroid inhibition of growth-related oncogene protein-alpha via mitogen-activated kinase phosphatase-1 in airway smooth muscle cells. J Immunol 2007; 178: 7366-7375.

29 Farias R, Rousseau S. The TAK1 $\rightarrow \mathrm{IKK} \beta \rightarrow \mathrm{TPL} 2 \rightarrow \mathrm{MKK} 1 / \mathrm{MKK} 2$ signaling cascade regulates IL-33 expression in cystic fibrosis airway epithelial cells following infection by Pseudomonas aeruginosa. Front Cell Dev Biol 2015; 3: 87.

30 Clark K, Plater L, Peggie M, et al. Use of the pharmacological inhibitor BX795 to study the regulation and physiological roles of TBK1 and IкB kinase epsilon: a distinct upstream kinase mediates Ser-172 phosphorylation and activation. J Biol Chem 2009; 284: 14136-14146.

31 Newton R, Holden NS, Catley MC, et al. Repression of inflammatory gene expression in human pulmonary epithelial cells by small-molecule IкB kinase inhibitors. J Pharmacol Exp Ther 2007; 321: 734-742.

$32 \mathrm{Wu} \mathrm{J}$, Powell F, Larsen NA, et al. Mechanism and in vitro pharmacology of TAK1 inhibition by (5Z)-7-oxozeaenol. ACS Chem Biol 2013; 8: 643-650.

33 Livak KJ, Schmittgen TD. Analysis of relative gene expression data using real-time quantitative PCR and the 2 $\left(-\Delta \Delta \mathrm{C}_{\mathrm{T}}\right)$ method. Methods 2001; 25: 402-408

34 Iwanaszko M, Kimmel M. NF- $\mathrm{KB}$ and IRF pathways: cross-regulation on target genes promoter level. $B M C$ Genomics 2015; 16: 307

35 Prefontaine D, Lajoie-Kadoch S, Foley S, et al. Increased expression of IL-33 in severe asthma: evidence of expression by airway smooth muscle cells. J Immunol 2009; 183: 5094-5103.

36 Dey N, Liu T, Garofalo RP, et al. TAK1 regulates NF- $\kappa$ B and AP-1 activation in airway epithelial cells following RSV infection. Virology 2011; 418: 93-101.

37 Pera T, Atmaj C, van der Vegt M, et al. Role for TAK1 in cigarette smoke-induced proinflammatory signaling and IL-8 release by human airway smooth muscle cells. Am J Physiol Lung Cell Mol Physiol 2012; 303: L272-L278.

38 Pera T, Sami R, Zaagsma J, et al. TAK1 plays a major role in growth factor-induced phenotypic modulation of airway smooth muscle. Am J Physiol Lung Cell Mol Physiol 2011; 301: L822-L828.

39 Long Q, Huang W, Yao Y, et al. Virus-like particles presenting interleukin-33 molecules: immunization characteristics and potentials of blocking IL-33/ST2 pathway in allergic airway inflammation. Hum Vaccin Immunother 2014; 10: 2303-2311. 